\title{
Phenotypic and Genetic Influences on Test-Day Measures of Acetone Concentration in Milk
}

\author{
G. M. Wood, ${ }^{1}$ P. J. Boettcher, ${ }^{1,2}$ D. F. Kelton, ${ }^{3}$ and G. B. Jansen ${ }^{1}$ \\ ${ }^{1}$ Department of Animal and Poultry Science and \\ ${ }^{2}$ Institute of Agricultural Biology and Biotechnology \\ National Research Council Segrate 20090 Italy. \\ ${ }^{3}$ Department of Population Medicine University of Guelph \\ Ontario N1G 2W1 Canada
}

\begin{abstract}
The objectives of this study were to estimate heritability of acetone concentration in milk, based on monthly samples of milk obtained as part of a routine milk testing program, and to evaluate the feasibility of using such data in a genetic evaluation program for selection against ketosis incidence. Milk samples were collected from January to December of 1999 in herds enrolled in the Ontario Dairy Herd Improvement Association, and acetone concentration was measured using an inline chemical procedure. The original data included more than 50,000 records. Because ketosis is generally a problem during early lactation, only the single test with the fewest days in milk was retained. In addition, data were retained only for cows with pedigree information. The final data set included 10,375 records. Among these data, only $6.56 \%$ had detectable levels of acetone. Acetone data were log-transformed prior to statistical analysis. Simple ANOVA indicated that herd, parity number, days in milk, and month of test had significant effects on acetone concentration. Acetone levels increased with lactation number and were higher in early lactation.

Three approaches were applied for genetic analysis. First, REML was used with a simple linear animal model. Then, a separate procedure used data augmentation and Gibbs Sampling to obtain continuously distributed underlying values for records with zero acetone concentration, and these data were analyzed with both an animal and sire model. Heritability of acetone concentration was less than $1 \%$ for all 3 analyses. Herd effects accounted for about $5 \%$ of the phenotypic variance. Low estimates of heritability were due either to low actual levels of genetic variance or inability to detect all of the genetic variance present, due to infrequent recording during the early part of the lactation.
\end{abstract}

Received July 17, 2003.

Accepted September 30, 2003.

Corresponding author: Paul Boettcher; e-mail: boettch@ibba.cnr.it.
Genetic evaluation based on recording of acetone concentration on a monthly basis seems of little use as a selection tool to decrease incidence of ketosis.

(Key words: ketosis, acetone, genetic evaluation)

\section{INTRODUCTION}

High-producing dairy cattle are subject to a number of health problems at and around the time of calving. The rapid rise in milk production early in lactation, coupled with a limited appetite, increases the metabolic load on the cow. Milk production is given metabolic priority over other physiological processes and nutrient intake may be insufficient to meet the increased demand for energy. Adipose tissues are thus mobilized in order to meet the metabolic needs of milk production. Nonesterified fatty acids are transported to the liver, where they are converted to glucose via the Kreb's cycle or to ketone bodies (acetone, acetoacetate, $\beta$-hydroxybutyrate) when oxaloacetate becomes limiting for the Kreb's cycle, which is often the case because feed consumption is depressed. Whereas excess acetone is easily eliminated via urine, milk, or expired air, acetoacetate can be detrimental to the health of the cow, leading to ketosis. Ketosis is generally accompanied by a loss of appetite, and milk production tends to decrease as the cow is unable to synthesize sufficient lactose due to the deficiency in glucose. Rapid loss of body condition often occurs (Baird, 1982).

Assuming that genetic factors affect incidence of ketosis, genetic evaluation of sires for incidence of ketosis in their daughters could provide a selection tool to decrease metabolic problems. However, some limitations to this possibility exist. First, few of the major dairy producing countries outside of Scandinavia have comprehensive recording plans for veterinary data upon which to base such a genetic evaluation, and the costs of establishing such a program would likely be high. Second, diagnosis of clinical ketosis is subjective, as different veterinarians may have different personal thresholds for declaring a cow clinically ketotic and prescribing treatment. In other cases, farmers may 
make the diagnosis and initiate treatment independently. In addition, ketosis exists in both clinical and subclinical forms and information about subclinical incidence would be useful for a genetic evaluation, but unavailable in veterinary data. For these reasons, an objective test for ketosis may yield more precision for a genetic evaluation of the disease (Emanuelson and Andersson, 1986).

Measuring acetone in milk has been shown to be a reliable method of estimating the incidence of hyperketonemia, or ketosis (Andersson, 1984). Ketone bodies are present in the milk at levels that are closely correlated $(r>0.8)$ with blood levels (Andersson, 1988). Acetone has the highest relative milk concentration and also shows the least diurnal variation, making it the best of the 3 ketone bodies to choose for ketosis testing (Andersson, 1988). Correlation between concentrations of acetone and acetoacetate are also quite high $(r=0.88$; Tveit et al., 1992).

A number of studies have estimated genetic heritabilities of concentrations of ketone bodies in milk (Emanuelson and Andersson, 1986; Gravert et al., 1991; Tveit et al., 1992), and results have been variable, ranging from near zero (Emanuelson and Andersson, 1986) to as high as 0.30 (Gravert et al., 1991). In contrast, estimates of heritabilities of ketosis incidence have ranged from around zero to about 0.10 (e.g., Gröhn et al., 1984; Mäntysaari et al., 1991). Previous studies on heritabilities of ketone bodies in milk were based on data collected from a few hundred to a few thousand cows. For genetic evaluation of cattle for level of acetone in milk, methods of mechanization of acetone testing must be used for easy capture of data. One such method has been developed in Denmark and was recently tested in Canada. Using this approach, acetone concentration in milk can be evaluated as part of the set of procedures now used to evaluate concentrations of fat, protein, and somatic cells in milk. However, questions remain about whether the sensitivity of this testing procedure will be high enough to provide genetic information usable for selection.

The objective of this study was to estimate parameters for environmental and genetic factors influencing concentrations of acetone in milk by using data from a national milk recording program, where acetone concentrations are recorded simultaneously with other components of milk. The purpose of the study was to determine whether such data could be reliably used for a national genetic evaluation of dairy sires for ketosis in their daughters.

\section{MATERIALS AND METHODS}

The data for this study were collected by the Ontario DHI corporation (Guelph, Ontario, Canada). Milk sam- ples collected on regular test days from registered Holstein cows in dairy herds in Southern Ontario were tested for milk acetone level using equipment provided by Foss Electric (Hillerød, Denmark). The acetone level was determined by a proprietary inline chemical procedure. Milk samples were tested over a 12 -mo period, beginning in January 1999 and ending in December of the same year. The acetone testing equipment was undergoing a 12-mo field trial during this period and, therefore, records prior to or after 1999 were not available. A total of 50,586 milk samples were tested for acetone level. To simplify the recording system, Ontario DHI did not directly record data for acetone concentration rather, samples were given a score to indicate the concentration of acetone in the milk. The following formula defines the relationship between test scores and concentrations of acetone:

$$
\mathrm{AS}=([\mathrm{A}]+0.10) \times 10,
$$

where AS is the acetone score recorded by the Ontario $\mathrm{DHI}$ and $[\mathrm{A}]$ is the acetone concentration $(\mathrm{mmol} / \mathrm{L})$. For example, a score of 1 corresponded to a concentration of $0 \mathrm{mmol} / \mathrm{L}$ of acetone in the milk, a score of 2 represented $0.1 \mathrm{mmol} / \mathrm{L}$ of acetone in the milk, and so forth.

The data were edited to exclude test-day records with daily milk production greater than $200 \mathrm{~kg}$, fat production greater than $9 \mathrm{~kg}$, or protein production greater than $6 \mathrm{~kg}$. A standard lactation length was imposed by deleting all records with DIM $<5$ or $>305$. Because genetic analyses were of interest, data from any cows without a registration number recorded were also deleted. The total number of records remaining after these edits was 40,182.

Pedigree data were provided by the Canadian Dairy Network (Guelph, Ontario, Canada). Registration numbers from the DHI records were matched with those in the provided database, which is used for the national genetic evaluations of dairy trait in Canada. Records for a given cow were eliminated from the set of acetone data if the registration number recorded in the DHI file could not be matched to a record in the national database. Of 11,716 unique cow registration numbers in the acetone data file, 10,375 could be successfully matched to the pedigree file. The final data set included 35,864 records from 10,375 cows sired by 1272 different bulls.

Because ketosis is primarily a problem in early lactation, only $4.00 \%$ of the tests showed any elevation in milk acetone. Therefore, to obtain a more accurate indication of presence of ketosis, only the acetone test with the lowest recorded DIM for each cow was used. This editing procedure increased the proportion of nonzero records to $6.56 \%$. Some records from later stages of 


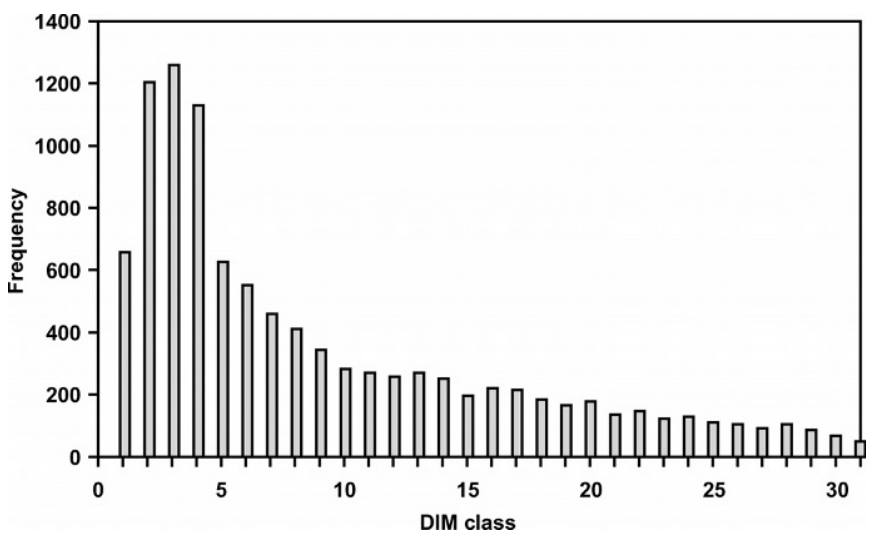

Figure 1. Distribution of acetone tests across DIM classes.

lactation remained because the 12 -mo trial period included cows whose lactations were already in progress at the start of the trial. Inclusion of these records allowed for the testing of effects of DIM across lactation. Not unexpectedly, the fact that records were taken from a single fixed-calendar-year period resulted in a skewed distribution of records per month. The number of tests used in the analysis was much higher in January and February than in the other months of the study. These records were from cows that did not initiate a new lactation in the remainder of the year. Slightly less than half of the data were recorded in these 2 mo and few tests $(<10 \%)$ were from the months of September to December. Due to the selection of the test with the lowest DIM, the number of tests was high in the early DIM classes and declined steadily as the DIM classes increased (Figure 1). Approximately two-thirds of the records were taken from within the first 100 DIM.

The distribution of raw acetone scores was highly skewed and thus data were transformed with natural logarithms as suggested by Emanuelson and Andersson (1986). The following linear model was initially applied to determine which nongenetic factors had significant influences on concentration of acetone in milk and were thus to be included in the subsequent genetic analyses:

$$
\begin{gathered}
\mathrm{y}_{\mathrm{ijkl}}=\mathrm{h}_{\mathrm{i}}+\mathrm{m}_{\mathrm{j}}+\mathrm{d}_{\mathrm{k}}+\mathrm{p}_{\mathrm{l}} \\
+(\mathrm{md})_{\mathrm{jk}}+(\mathrm{mp})_{\mathrm{jl}}+(\mathrm{dp})_{\mathrm{kl}}+\mathrm{e}_{\mathrm{ijklm}},
\end{gathered}
$$

where $\mathrm{y}_{\mathrm{ijkl}}$ is the mth log transformed acetone score from the ith herd, the jth month, the kth DIM class, and the lth lactation; $h_{i}$ is the fixed effect of the ith herd ( $i=1, \ldots, 184) ; m_{j}$ is the fixed effect of the jth test month $(j=1, \ldots, 12) ; d_{k}$ is the fixed effect of the kth DIM class $(\mathrm{k}=1, \ldots, 31) ; \mathrm{p}_{\mathrm{l}}$ is the fixed effect of the $\mathrm{pth}$ parity $(1,2,3$, and $\geq 4) ;(\mathrm{md})_{\mathrm{jk}},(\mathrm{mp})_{\mathrm{jl}}$, and $(\mathrm{dp})_{\mathrm{kl}}$ are fixed effects of 2-way interactions among month, DIM, and parity; and $\mathrm{e}_{\mathrm{ijklm}}$ is the random residual effect for the corresponding record. Days in milk were defined with 31 classes of 10-d intervals (except the 31st class, which corresponded to DIM 300 to 305). Statistical significance of the various factors was tested with ANOVA.

Three genetic analyses were applied. The first approach applied a simple linear model to the transformed scores, despite their nonnormal distribution. Estimates of genetic parameters were based on REML and were obtained using the VCE software of Groeneveld (1996). The model equation for this analysis was similar to that applied for estimation of significance of fixed effects:

$$
\mathrm{y}_{\mathrm{ijklm}}=\mathrm{h}_{\mathrm{i}}+\mathrm{m}_{\mathrm{j}}+\mathrm{d}_{\mathrm{k}}+\mathrm{p}_{\mathrm{l}}+\mathrm{a}_{\mathrm{m}}+\mathrm{e}_{\mathrm{ijklm}} .
$$

Factors in the model were the same as in Equation [2], except that the random effect of animal $\mathrm{m}\left(\mathrm{a}_{\mathrm{m}}\right)$ was also considered. In addition, herd effects $\left(h_{i}\right)$ were considered to be random, rather than fixed. Herd effects were considered random because no direct selection for acetone level could have taken place across herds. Thus no confounding of herd effects and genetic levels of acetone was expected. The pedigree file used for this analysis included 24,294 animals and was obtained by tracing relationships back for 3 generations. All unknown parents were assumed to be from the same base population.

The second type of genetic analysis used data augmentation and Gibbs sampling to account for the nonnormal distribution of the acetone scores. This approach combined aspects of linear and threshold models. The analysis assumed that variability in resistance to ketosis existed on an underlying normal scale for all animals, including those with nonmeasurable concentrations of acetone in their milk (score $=1$ ), but that this variability was unobserved until the underlying value exceeded a given threshold $($ score $=2)$. For this analysis, data from animals with scores corresponding to detectable levels of acetone (i.e., acetone score $>2$ ) were used directly. For each animal with no measurable acetone concentration (score $=1$ ) an underlying value was generated by sampling from a truncated normal distribution, using the data augmentation procedure described by Sorensen (1999). In this procedure, new underlying scores were generated in each round of Gibbs sampling according to the following equation:

$$
\mathrm{u}_{\mathrm{ijklm}}{ }^{\mathrm{r}}=\mathrm{h}_{\mathrm{i}}^{\mathrm{r}-1}+\mathrm{m}_{\mathrm{j}}^{\mathrm{r}-1}+\mathrm{d}_{\mathrm{k}}^{\mathrm{r}-1}+\mathrm{p}_{\mathrm{l}}^{\mathrm{r}-1}+\mathrm{a}_{\mathrm{m}}^{\mathrm{r}-1}+\mathrm{e}_{\mathrm{ijklm}}{ }^{\mathrm{r}},
$$

where $\mathrm{u}_{\mathrm{ijklm}}{ }^{\mathrm{r}}$ is the underlying transformed acetone score for the rth round of Gibbs sampling for the transformed acetone score of the mth animal; $h_{i}{ }^{r-1}$ is the solution for the ith herd effect in the (r-1)th round of 


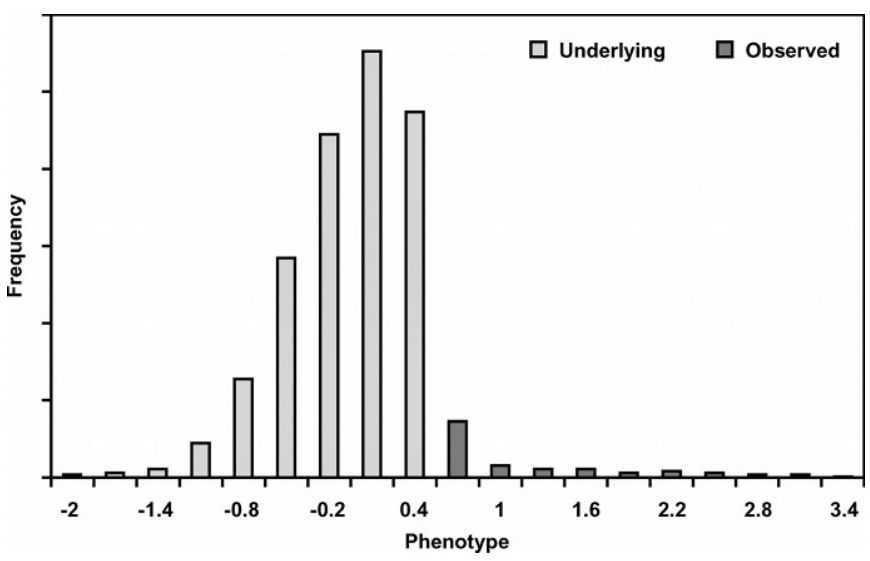

Figure 2. Typical distribution of underlying and observed phenotypes analyzed with the linear-threshold model.

Gibbs sampling; $\mathrm{t}_{\mathrm{j}}^{\mathrm{r}-1}, \mathrm{~d}_{\mathrm{k}}^{\mathrm{r}-1}, \mathrm{l}_{\mathrm{l}}^{\mathrm{r}-1}$, and $\mathrm{a}_{\mathrm{m}}^{\mathrm{r}-1}$ are corresponding values for effects of month, DIM, parity, and animal; and $\mathrm{e}_{\mathrm{ijk} \mathrm{km}}{ }^{\mathrm{r}}$ is a randomly drawn value from a distribution $\mathrm{N}\left(0,\left[\sigma_{\mathrm{e}}^{2}\right]^{\mathrm{r}-1}\right)$, where $\left[\sigma^{2} \mathrm{e}\right]^{\mathrm{r}-1}$ is the sampled value for residual variance from the $r-1$ th round of Gibbs sampling. The distribution of $\mathrm{u}_{\mathrm{ijklm}}{ }^{\mathrm{r}}$ was truncated at a maximum of 0.6931 (the minimum value for animals with detectable levels of acetone in milk), so resampling was performed when necessary to obtain values less than this threshold. Figure 2 shows an example of the distribution of underlying and observed obtained using the described data augmentation procedure. As can be seen from Figure 1, this procedure resulted in a distribution of values that was nearly normal but with a long right tail.

Real and sampled underlying scores were then combined and analyzed with a linear model according to Equation [3]. This procedure was similar to the method proposed by Korsgaard et al. (2003) to simultaneously evaluate observed and censored data for a failure timebased trait. For implementation of the Gibbs sampler, effects for herd, month, DIM, parity, and animal were set to zero in the first round and then drawn from normal distributions in subsequent cycles. Variances were drawn from inverted chi-square distributions. Prior values for variances were chosen based on results from the linear model. Two chains yielding a total of 600,000 rounds (after burn-in) were used to obtain posterior distributions for the variances of interest.

Application of a threshold model with an animal model has been shown to often produce spurious results (e.g., Hoeschele and Tier, 1995; Luo et al., 2001). For this reason, the proposed combined linear-threshold model was also applied using a sire model. This sire model was implemented in the same way as the animal model and effects included were the same as in
Equation [3], except that the animal effect was replaced by a sire effect. Also, sires were required to have at least 3 offspring, which reduced the data set to 10,114 records. Because the variability of estimates for variance components is greater for a sire model than for an animal model, 2 chains yielding 1,000,000 total samples were used to establish posterior distributions of the sire model.

Although they were more complicated to implement, the 2 linear-threshold models were expected to be more statistically appropriate than the simple linear model and yield estimates of heritabilities on the underlying liability scale rather than the observed scale. As such, estimates of heritabilities were expected to be somewhat greater than those from the linear model, according to theory (Gianola, 1982) and consistent with previous work comparing linear and threshold models (e.g., Luo et al. 2001).

\section{RESULTS}

The overall mean of the 10,375 milk acetone scores was 1.227 , which corresponds to a mean concentration of $0.0227 \mathrm{mmol} / \mathrm{L}$. The minimum test value was $1.0(0.0$ $\mathrm{mmol} / \mathrm{L})$ and the maximum was $49(4.8 \mathrm{mmol} / \mathrm{L})$. The standard deviation was 1.54 . As mentioned previously, fewer than $10 \%$ of the scores indicated measurable levels of acetone, so both the median and mode of the distribution of scores were 1.0 , or $0.0 \mathrm{mmol} / \mathrm{L}$.

The means and other descriptive statistics for each parity are summarized in Table 1 . The proportion of cattle having an acetone concentration $>0.0 \mathrm{mmol} / \mathrm{L}$ increased with each lactation. The proportion of cattle in lactation 4 or greater exceeding $0.0 \mathrm{mmol} / \mathrm{L}(9.08 \%)$ was approximately twice as high as the proportion in first parity $(4.50 \%)$. The mean acetone concentration in second lactation was approximately the same as in first lactation, but more second-lactation cattle had detectable levels of acetone in their milk.

Results from the ANOVA for nongenetic effects on acetone concentration (log of acetone score) are in Table 2. The $R^{2}$ of the analysis of transformed scores was 0.102. All of the main factors had highly significant effects, and $P$ values for all factors but month were < 0.0001. Two-way interactions among effects were not important, however. For this reason, all of the main factors, but no interactions, were included in subsequent genetic analyses.

Least square means were obtained for the nongenetic factors. These results are presented in Table 3 for parities and months of test. As had been indicated by the simple descriptive statistics in Table 2, acetone scores were higher in later lactations ( 3 and 4+) than in lactations 1 and 2 . Although differences among months were 
Table 1. Descriptive statistics for acetone concentration $(\mathrm{mmol} / \mathrm{L})$ and corresponding $\operatorname{scores}^{1}$ (in parentheses) for different lactations.

\begin{tabular}{llllll}
\hline Lactation & $\mathrm{n}$ & $\%>0$ & Mean & $\mathrm{SD}$ & Maximum $^{2}$ \\
\hline 1 & 3464 & 4.50 & $0.014(1.14)$ & $0.10(1.04)$ & $2.1(22.0)$ \\
2 & 2730 & 5.97 & $0.014(1.14)$ & $0.10(0.99)$ & $2.2(23.0)$ \\
3 & 1858 & 8.13 & $0.032(1.32)$ & $0.19(1.94)$ & $4.0(41.0)$ \\
$>4$ & 2323 & 9.08 & $0.038(1.37)$ & $0.22(2.18)$ & $4.8(49.0)$ \\
\hline
\end{tabular}

${ }^{1}$ Acetone score $=[$ Acetone concentration +0.10$] \times 10$.

${ }^{2}$ Minimum concentration was zero for all lactations.

significant, no obvious pattern in the least square means was observed (Table 3). However, results suggested that acetone concentrations tend to be lower during the winter months, as October, November, December, and January had the 4 lowest least square means. Acetone levels were highest in April. Figure 3 shows the trend in acetone levels (based on least square means for log acetone score) for different DIM. As expected, due to the higher incidence of ketosis in early lactation, the least square means were highest in the early DIM classes and dropped sharply through the first 6 DIM classes. The least square mean for the first period, corresponding to $\mathrm{d} 5$ to 14 , was more than 5 times greater than the least square mean for the sixth period (d 55 to 64). The overall trend in acetone levels was relatively flat after about d 85, with minor oscillation likely due only to sampling variability.

Estimates of heritabilities and proportions of variance due to herd effects are in Table 4. Heritability for acetone level was very low, averaging less than $0.5 \%$ for the 3 analyses. Estimates were higher for the linearthreshold models than from the linear model, similar to results observed in previous studies comparing full threshold and linear models (e.g., Luo et al., 2001). In all cases, the estimate of heritability was no more than 2 times the corresponding measure of variability for the estimate. The maximum estimate was 0.0089 , based on the linear-threshold sire model. Herd effects accounted for about 3 to $5 \%$ of the variance in acetone level, depending on the statistical analysis used. These results demonstrate that differences across herds were a much more important factor influencing acetone level in milk than were genetic effects. Emanuelson and Andersson

Table 2. The ANOVA for effects of nongenetic factors on acetone scores.

\begin{tabular}{lrcc}
\hline Source & df & $F$ value & $\operatorname{Pr}>F$ \\
\hline Model & 227 & 5.10 & $<0.0001$ \\
Herd & 183 & 3.00 & $<0.0001$ \\
DIM class & 30 & 11.23 & $<0.0001$ \\
Lactation & 3 & 20.99 & $<0.0001$ \\
Test month & 11 & 2.67 & 0.002 \\
\hline
\end{tabular}

(1986) had previously reported that contemporary group (herd-season in their study) was the most important source of variation for acetone measures.

In a final verification step, a fourth genetic analysis was performed. For this analysis, data were edited to include only those records within the first $100 \mathrm{~d}$ of lactation. This approach of editing concentrated on those DIM during which acetone was most likely to be elevated. In fact, among the 7558 records remaining under this criterion, $8.78 \%$ of records showed nonzero values for acetone, compared with $6.56 \%$ in the set without a restriction on DIM. The linear-threshold animal model was applied, using a pedigree file containing 18,018 animals. This analysis resulted in a somewhat higher, but still very low, estimate of heritability of 0.005 .

\section{DISCUSSION}

Several possible reasons can be proposed to explain the low estimates of heritability for milk acetone concentration. First, the trait may simply be under minimal genetic control. This explanation is supported by the findings of Emanuelson and Andersson (1986), who also obtained estimates for heritability for milk acetone concentration that were low and not significantly different from zero. Estimates of heritability for clinical ketosis have also been low (Gröhn et al., 1984; Mäntysaari

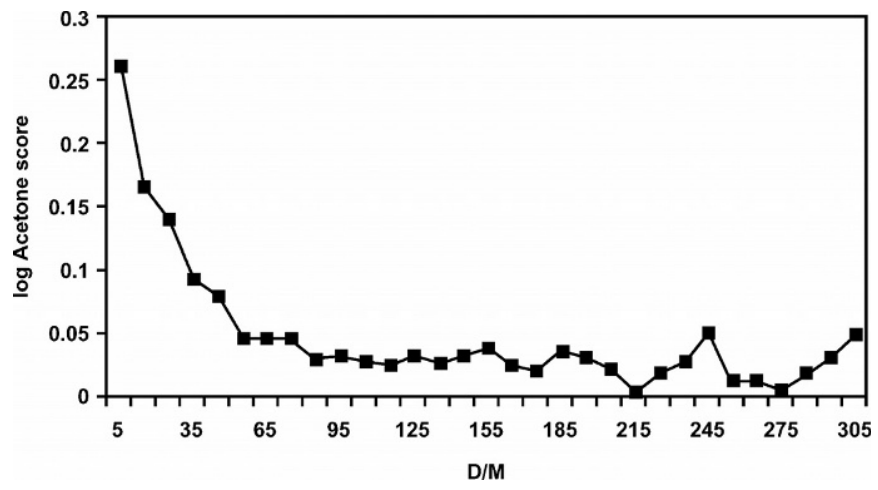

Figure 3. Least square means of acetone scores (log transformed) across DIM. 
Table 3. Least square means of log transformed acetone scores for different lactations and different months.

\begin{tabular}{ll}
\hline Effect & Log score \\
\hline Lactation 1 & 0.018 \\
Lactation 2 & 0.027 \\
Lactation 3 & 0.064 \\
Lactation $>4$ & 0.080 \\
January & 0.024 \\
February & 0.052 \\
March & 0.038 \\
April & 0.097 \\
May & 0.055 \\
June & 0.068 \\
July & 0.077 \\
August & 0.045 \\
September & 0.059 \\
October & 0.011 \\
November & 0.025 \\
December & 0.013 \\
\hline
\end{tabular}

et al., 1991). However, the results of Gravert et al. (1991) suggested that acetone concentration in milk might be under moderate genetic control.

Another explanation for the low estimates of heritability could be that the system used for data collection may not be sufficient for capturing all of the genetic variability that exists within the population. Sensitivity of the equipment did not seem to be a limiting factor. The maximum value was many times greater than the minimum nonzero value, so increasing the sensitivity of the equipment to obtain more precise scores between zero and the minimum nonzero value observed in this study would likely have been of little value. The more serious limitation of the recording system was probably associated with the timing and frequency of tests, particularly in early lactation. With monthly tests, acetone level was recorded only about every 25 to $45 \mathrm{~d}$ for each cow. In addition, the design of the study, with a short, 1-yr period of data collection, may have contributed, as some cows were tested only during the latter part of lactation. Such a recording scheme resulted in a small proportion $(<20 \%)$ of cows being tested during the first 2 to 3 wk after calving, the critical period for ketosis (Figure 1). Thus, many cows may have had elevated acetone levels during periods when no tests were taken and then returned to normal levels prior to the subsequent sample. Only about $6.5 \%$ of the cows showed any elevation in acetone concentration, which was far lower than the $25 \%$ prevalence of subclinical ketosis observed by Duffield et al. (1998), who collected samples within the first 2 wk after parturition instead of on regular DHI test days. The milk acetone tests based on monthly milk samples do not appear to give an accurate representation of the number of animals with elevated ketone body levels.

\section{CONCLUSIONS}

Estimates of heritability for acetone concentration in milk were very low $(<0.01)$. The low estimate of heritability may have been due either to low natural genetic variability for the trait or to limitations in the recording system, linked to a relative scarcity of observations during the early weeks of lactation. Regardless of the reason for the low heritability, these results suggest that a genetic evaluation based on test-day samples of acetone concentration is unlikely to yield valuable information for selection against ketosis. Additional research would be needed to determine whether more frequent measures of acetone concentration yield higher estimates of heritability and whether the expense of routine collection of such samples outside of existing DHI could be cost efficient. Monthly tests of acetone concentration may nevertheless be of use for management purposes. Although such a testing scheme will not detect all cows with subclinical ketosis, at least a portion of such cows could be identified and those cows could be treated, especially if they also showed other indications of subclinical ketosis, such as abnormally high fat-to-protein ratio in their milk. Also, herd managers could use such data to compare the average incidence of elevated acetone in their respective herds with population means or other guidelines and adjust management practices, if necessary, to reduce incidence of ketosis.

Table 4. Proportions of variance in log transformed acetone scores due to genetic (heritability) and herd effects, according to various statistical analyses.

\begin{tabular}{|c|c|c|c|c|}
\hline \multirow[b]{3}{*}{ Analysis } & \multicolumn{4}{|c|}{ Effect } \\
\hline & \multicolumn{2}{|c|}{ Genetic } & \multicolumn{2}{|c|}{ Herd } \\
\hline & Estimate $^{1}$ & Dispersion & Estimate & Dispersion \\
\hline Linear animal model & 0.0020 & 0.0040 & 0.0370 & 0.0040 \\
\hline Linear-threshold animal model & 0.0033 & 0.0016 & 0.0530 & 0.0096 \\
\hline Linear-threshold sire model & 0.0089 & 0.0046 & 0.0550 & 0.0099 \\
\hline
\end{tabular}

${ }^{1}$ Posterior mean for linear-threshold models. 


\section{ACKNOWLEDGMENTS}

The authors would like to thank the Ontario Ministry of Agriculture and Food and the Dairy Farmers of Ontario for supporting this project financially and Foss Electric for supplying the equipment used to obtain measures of acetone concentration. We also recognize the Ontario Dairy Herd Improvement Corporation and the Canadian Dairy Network for their contribution of the data used in this study.

\section{REFERENCES}

Andersson, L. 1984. Concentrations of blood and milk ketone bodies, blood isopropanol, and plasma glucose in dairy cows in relation to the degree of hyperketonaemia and clinical signs. Zbl. Vet. Med. 31:683-693.

Andersson, L. 1988. Subclinical ketosis in dairy cows. Vet. Clin. North Am. Food Anim. Pract. 4:233-251.

Baird, G. D. 1982. Primary ketosis in the high-producing dairy cow: Clinical and subclinical disorders, treatment, prevention, and outlook. J. Dairy Sci. 65:1-10.

Duffield, T. F., D. Sandals, K. E. Leslie, T. K. Lissemore, B. W. McBride, J. H. Lumsden, P. Dick, and R. Bagg. 1998. Efficacy of Monensin for the prevention of subclinical ketosis in lactating dairy cows. J. Dairy Sci. 81:2866-2873.
Emanuelson, U., and L. Andersson. 1986. Genetic variation in milk acetone in Swedish dairy cows. J. Vet. Med. 33:600-608.

Gianola, D. 1982. Theory and analysis of threshold characters. J. Anim. Sci. 63:217-244.

Gravert, H. O., E. Hensen, H. Hafezian, K. Pabst, and H. SchulteCoerne. 1991. Umweltbedingte und genetische Einflusse auf den Acetongehalt der Milch. Zuchtungskunde 63:42-50.

Gröhn, Y. T., J. R. Thompson, and M. L. Bruss. 1984. Epidemiology and genetic basis of ketosis in Finnish Ayrshire cattle. Prev. Vet. Med. 3:65-77.

Hoeschele, I., and B. Tier. 1995. Estimation of variance components of threshold characters $\mathrm{b}$ marginal posterior modes and means via Gibbs sampling. Genet. Sel. Evol. 27:519-540.

Korsgaard, I. R., M. S. Lund, D. Sorensen, D. Gianola, P. Madsen, and J. Jensen. 2003. Multivariate Bayesian analysis of Gaussian, right censored Gaussian, ordered categorical, and binary traits using Gibbs sampling. Genet. Sel. Evol. 35:159-183.

Luo, M. F., P. J. Boettcher, L. R. Schaeffer, and J. C. M. Dekkers, 2001. Bayesian inference for categorical traits with an application to variance component estimation. J. Dairy Sci. 84:694-704.

Mantysaari, E. A., Y. T. Gröhn, and R. L. Quaas. 1991. Clinical ketosis: Phenotypic and genetic correlations between occurrences and with milk yield. J. Dairy Sci. 74:3985-3993.

Sorensen, D. 1999. Gibbs Sampling in Quantitative Genetics. Danish Institute of Agricultural Sciences. Tjele, Denmark.

Tveit, B., F. Lingaas, M. Svendsen, and O. V. Sjaastad. 1992. Etiology of acetonemia in Norwegian cattle I. Effect of ketogenic silage, season, energy level, and genetic factors. J. Dairy Sci. 75:24212432 